\title{
Intelligent Training in Control Centres Based on an Ambient Intelligence Paradigm
}

\author{
Luiz Faria ${ }^{1}$, António Silva ${ }^{1}$, Carlos Ramos ${ }^{1}$, Zita Vale $^{1}$, and Albino Marques ${ }^{2}$ \\ ${ }^{1}$ GECAD - Knowledge Engineering and Decision Support Group / Institute of \\ Engineering - Polytechnic of Porto, Portugal \\ \{lef, ass, csr, zav\} @isep.ipp.pt \\ ${ }^{2}$ REN - Transmission Network, Portugal \\ albino.marquesaren.pt
}

\begin{abstract}
This article describes a new approach in the Intelligent Training of Operators in Power Systems Control Centres, considering the new reality of Renewable Sources, Distributed Generation, and Electricity Markets, under the emerging paradigms of Cyber-Physical Systems and Ambient Intelligence. We propose Intelligent Tutoring Systems as the approach to deal with the intelligent training of operators in these new circumstances.
\end{abstract}

Keywords: Ambient intelligence, Artificial intelligence, Collaborative work, Cyber-physical systems, Intelligent tutoring systems, Power system restoration, SCADA systems.

\section{Introduction}

Traditionally, Power Systems were conceived to provide electrical energy under security conditions. Guaranteeing sustainable development is a huge challenge for Power Systems. This requires a significant increasing in Distributed Generation, mainly based on renewable sources. However, this leads to a system that is much more complex to control, since we have many more Power Generation plants, and the generation is more unpredictable than before, due to the difficulty in forecasting the energy production in some renewable sources (e.g. wind and photovoltaic).

The introduction of Electricity Markets, show us the fragility of Power Systems infrastructures. Several severe incidents, including blackouts, occurred (e.g. the 14th August 2003 Blackout in USA, and the 4th October 2006 quasi-blackout affecting 9 European countries). However, the problem is more human than technical, since some of these incidents were caused or had increased consequences due to operators' mistakes, namely at the level of Power Systems Control Centers (CC)[1]. There is a trend to the degradation of this situation with the increasing in the complexity of Power Systems due to the augmenting number of Renewable source installations in the near future.

Thus, training Power Systems Control Centres' Operators for this new reality is a critical goal. For this purpose we are developing CITOPSY (Cyber-Ambient Intelligent Training of Operators in Power Systems CC) project. The project considers the following emerging and advanced paradigms: 
- Cyber-Physical Systems (CPS), since Power Systems networks, strongly based on SCADA systems, that are expected to evolve for Intelligent SCADA, are typically pointed as a reference example of CPS;

- Ambient Intelligence (AmI) [2], since we claim that CC are a very good example of environment where AmI makes sense;

- and Intelligent Tutoring System (ITS), an advanced technology that is now achieving the maturity to be used in real-world applications, and that is specially adequate for training, namely when combined with simulators. Here we have some experience in the development of ITS for Power Systems incident analysis and diagnosis, power restoration, according to an integrated view [3].

\section{Background}

In this section we will describe the following aspects: Cyber-Physical Systems (CPS); Ambient Intelligence (AmI); and Intelligent Tutoring Systems (ITS). These paradigms and technologies will be seen in accordance with the Power Systems Control Centres perspective and under the new Electricity Markets and Renewable Sources reality.

CPS are computing systems interacting with physical processes. CPS are typically designed as networks of interacting elements instead of as standalone devices [4]. CPS use computations and communication deeply embedded in and interacting with physical processes to add new capabilities to physical systems [5]. CPS must be dependable, secure, safe, efficient, and operate in real-time. They must also be scalable, cost-effective and adaptive (http://varma.ece.cmu.edu/cps/CFP.htm). The integration of computational and physical processes exhibits a complex behaviour that cannot be analyzed by the computational or physical sciences alone. These systems also transcend traditional computer-controlled systems because of their scale, dependence on man-machine interaction and their rich communication infrastructure that is enabled by the Internet (http://www.qhdctc.com/wcps2008/center.htm).

CPS range from miniscule (pace makers) to large-scale (the national powergrid, blackout-free electricity generation and distribution, optimization of energy consumption) [5].

Power Networks' critical physical infrastructure depends crucially on SCADA (Supervisory Control and Data Acquisition) and DCS (Digital Control Systems) for sensing, monitoring, gathering, and control of distributed physical infrastructures. Power Systems CC are the place where all the information of SCADA and DCS arrive, and CC Operators' must handle the huge amount of data and information arriving from these systems, namely when we are in the presence of critical incidents. Now Cyber-Physical Intelligence is emerging as an important sub-area. The concept of Intelligent SCADA, with decentralized, flexible, and intelligent behavior, being dynamically adaptive to the context of the Power System is appearing [6].

Ambient Intelligence (AmI) can be seen as a digital environment that proactively, but sensibly, supports people in their daily lives [7]. The European Commission's IST Advisory Group - ISTAG believes that it is necessary to take a holistic view of Ambient Intelligence (AmI), considering not just the technology, but the whole of the innovation supply-chain from science to end-user, and also the various features of the 
academic, industrial and administrative environment that facilitate or hinder realisation of the AmI vision [8].

Most of the work developed today in AmI deals with environments like home, offices, hospitals, cars, transportation systems, museums, and cultural heritage [9]. In [2] authors point to $\mathrm{CC}$ like one of the tentative environments where the Ambient Intelligence will be used in the future.

ITS are able to instruct and train students and professionals without the intervention of human beings [10]. ITS introduce a set of ideas like the domain knowledge representation, allowing the possibility to reason and explain automatically on domain problems. Developments were made in trainees' models, instructional and pedagogical planning, and user interface. Some ITS ideas were incorporated in other computeraided instruction paradigms, like e-learning and distributed learning. However there is a clear difference since these learning concepts are more centered in the interaction with the instructor. Today, ITS can be produced by authoring tools [11], and specific evaluation and assessment methods can be used, appearing sometimes in the form of Cognitive Tutors [12].

ITS were applied in many domains, from medicine to computer programming. There is a set of innovative applications of ITS for training, even in critical and complex domains. Some of these applications provide on-the-job training and can be combined with domain simulators [13].

Electricity Markets bas been a challenge for Power Systems \& Economics scientific communities [14]. They introduced a new degree of complexity in Power System Networks control. On the other hand, for environmental reasons most of the new generation plants are based on Renewable Sources of Energy, for which production forecasting is more difficult to perform. This new Distributed Generation scenario points out for an urgent need to create a new generation of tools for training $\mathrm{CC}$ operators, combining technologies like ITS, AmI, and CPS.

\section{An Intelligent Tutoring System for the Diagnosis and Restoration of Power Systems}

The main goal of this work is to supply CC operators with an intelligent environment, fully integrated with the SCADA system, and to provide the CC room with training abilities. In order to accomplish this goal, we will proceed with the integration of an ITS for the diagnosis and restoration of Power Systems in a CC room.

\subsection{Tutoring Environment Architecture}

Fig. 1 shows this tutoring environment architecture, composed of three complex systems: a CPS module responsible for the acquisition and treatment of the network's physical data; an AmI system (Interaction Manager) that allows the trainee's immersion in the control room environment; and a tutoring system responsible for the pedagogical process. The tutoring system involves two main areas: one devoted to the training of fault diagnosis skills and another dedicated to the training of power system restoration techniques. 


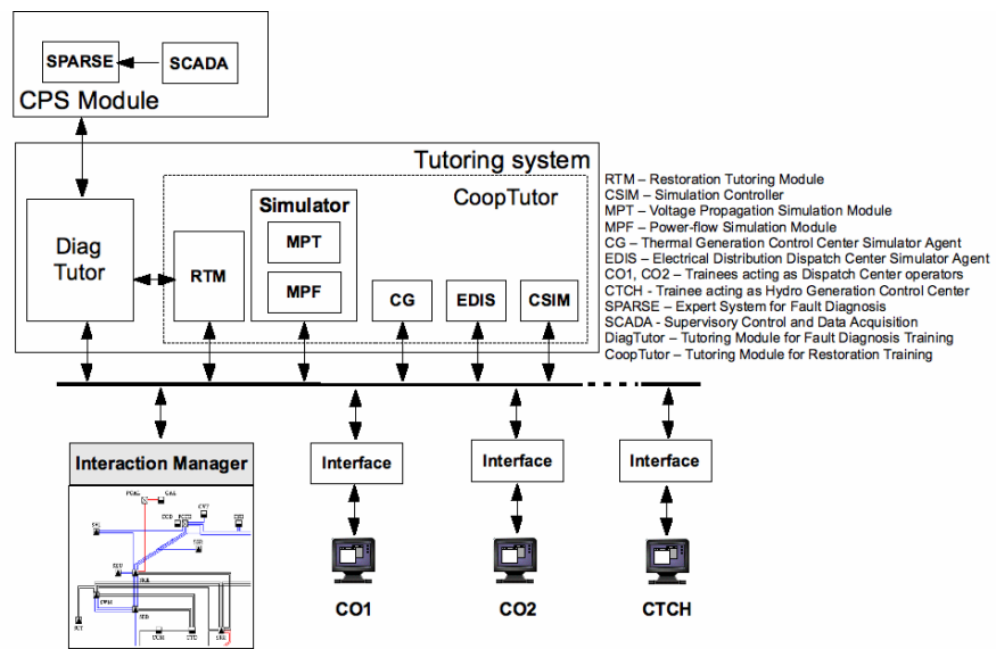

Fig. 1. Tutoring Environment Architecture

The selection of the adequate established restoration procedure strongly depends on the correct identification of the Power System operation state. Therefore, the identification of the incidents or set of incidents occurring in the transmission network is of utmost relevance in order to establish the current Power System operation state. Thus the proposed training framework divides operator's training in to distinct stages. The first one, as described in session 3.2, is intended to give operators with competence needed to get incident diagnosis. After that, operators are able to use CoopTutor to train their skills to manage the restoration procedures (session 3.3).

\subsection{Tutoring Module for Fault Diagnosis Training}

During the analysis of lists of alarm messages, CC operators must have in mind the group of messages that describes each type of fault. The same group of messages can show up in the reports of different types of faults. So $\mathrm{CC}$ operators have to analyze the arrival of additional information, whose presence or absence determines the final diagnosis.

Operators have to deal with uncertain, incomplete and inconsistent information, due to data loss or errors occurred in the data gathering system.

The interaction between the trainee and the tutor is performed through prediction tables where the operator selects a set of premises and the corresponding conclusion. The premises represent events (SCADA messages), temporal constraints between events or previous conclusions.

DiagTutor does not require the operator's reasoning to follow a predefined set of steps, as in other implementations of the model tracing technique [15]. In order to evaluate this reasoning, the tutor will compare the prediction tables' content with the specific situation model. This model is obtained by matching the domain model with the inference undertaken by SPARSE expert system [1]. This process is used to: identify the errors revealing operator's misconceptions; provide assistance on each 
problem solving action, if needed; monitor the trainee knowledge evolution; and provide learning opportunities for the trainee to reach mastery. In the area of ITS's this goals has been achieved through the use of cognitive tutors [12].

The identified errors are used as opportunities to correct the faults in the operator's reasoning. The operator's entries in prediction tables cause immediate responses from the tutor. In case of error, the operator can ask for help which is supplied as hints. Hinting is a tactic that encourages active thinking structured within guidelines dictated by the tutor. The first hints are generic, becoming more detailed if the help re-quests are repeated.

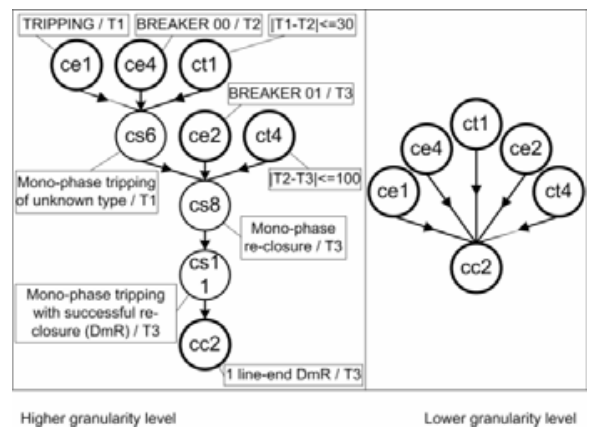

Fig. 2. Higher and lower granularity levels of the situation specific model

The situation specific model generated by the tutoring system for the problem presented is shown in the left frame of Fig. 2. It presents high granularity since it includes all the elementary steps used to get the problem solution. The tutor uses this model to detect errors in the operator reasoning by comparing the situation specific model with the set of steps used by the operator. This model's granularity level is adequate to a novice trainee but not to an expert operator. The right frame of Fig. 2 represents a model used by an expert operator, including only concepts representing events, temporal constraints between events and the final conclusion. Any reasoning model between the higher and lower granularity level models is admissible since it does not include any violation to the domain model. These two levels are used as boundaries of a continuous cognitive space.

Indeed, the process used to evaluate the trainee's reasoning is based on the application of pattern-matching algorithms. Similar approaches with the same propose are used in other ITS's, such as in the TAO ITS [16], an ITS designed to provide tactical action officer students at US navy with practice-based and individualized instruction.

\subsection{Tutoring Module for Restoration Training}

The management of a power system involves several distinct entities, responsible for different parts of the network. The power system restoration needs a close coordination between generation, transmission and distribution personnel and their actions should be based on a careful planning and guided by adequate strategies [17]. 
In the specific case of the Portuguese transmission network, four main entities can be identified: the National Dispatch Center (CC), responsible for the energy management and for the thermal generation; the Operational Centre $(\mathrm{CO})$, controlling the transmission network; the Hydroelectric Control Centers (CTCH), responsible for the remote control of hydroelectric power plants and the Distribution Dispatch (EDIS), controlling the distribution network.

The power restoration process is conducted by these entities in such a way that the parts of the grid they are responsible for will be slowly led to their normal state, by performing the actions specified in detailed operating procedures and fulfilling the requirements defined in previously established protocols. This process requires frequent negotiation between entities, agreement on common goals to be achieved, and synchronization of the separate action plans on well-defined moments.

Several agents personify the four entities that are present in the power system restoration process. This multi-agent approach was chosen because it is the most natural way of translating the real-life roles and the split of domain knowledge and performed functions that can be witnessed in the actual power system. Several entities responsible for separate parts of the whole task must interact in a cooperative way towards the fulfillment of the same global purpose. Agents' technology has been considered well suited to domains where the data is split by distinct entities physically or logically and which must interact with one another to pursue a common goal [18].

In this section we describe how we developed a training environment able to deal adequately with the training of the procedures, plans and strategies of the power system restoration, using what may be called lightweight, limited scope simulation techniques. This environment's purpose is to make available to the trainees all the knowledge accumulated during years of network operation, translated into detailed power system restoration plans and strategies, in an expedite and flexible way. The embedded knowledge about procedures, plans and strategies should be easily revisable, any time that new field tests, post-incident analysis or simulations supply new data.

These agents can be seen as virtual entities that possess knowledge about the domain. As real operators, they have tasks assigned to them, goals to be achieved and beliefs about the network status and others agents' activity. They work asynchronously, performing their duties simultaneously and synchronizing their activities only when this need arises. Therefore, the system needs a facilitator (simulator in Fig. 1) that supervises the process, ensuring that the simulation is coherent and convincing.

In our system, the trainee can choose to play any of the available roles, namely the $\mathrm{CO}$ and the $\mathrm{CC}$ ones, leaving to the tutor the responsibility of simulating the other participants.

The ITS architecture was planned in order that future upgrades of the involved entities or the inclusion of new agents to be simple.

The representation method used to model the trainee's knowledge about the domain knowledge is a variation of the Constraint-Based Modeling (CBM) technique [19]. This student model representation technique is based on the assumption that diagnostic information is not extracted from the sequence of student's actions but rather from the situation, also described as problem state, that the student arrived at. Hence, the student model should not represent the student's actions but the effects of these actions. Because the space of false knowledge is much greater than the one for the correct one, it was suggested the use of an abstraction mechanism based on 
constraints. In this representation, a state constraint is an ordered pair $(\mathrm{Cr}, \mathrm{Cs})$ where $\mathrm{Cr}$ stands for relevance condition, and Cs for satisfaction condition. $\mathrm{Cr}$ identifies the class of problem states in which this condition is relevant and Cs identifies the class of relevant states that satisfy Cs. Under these assumptions, domain knowledge can be represented as a set of state constraints. Any correct solution for a problem cannot violate any of the constraints. A violation indicates incomplete or incorrect knowledge and constitutes the basic piece of information that allows the Student Model to be built on.

This CBM technique does not require an expert module and is computationally undemanding because it reduces student modeling processing to a basic pattern matching mechanism [20]. One example of a state constraint, as used in our system, can be found below:

If there is a request to CTCH to restore the lines under its responsibility

Then the lines that connect to the hydroelectric power plants must already have been restored

\section{Otherwise an error has occurred}

Each violation to a state constraint like the one above enables the tutor to intervene both immediately or at a later stage, depending on the seriousness of the error or the pedagogical approach that was chosen.

This technique gives the tutor the flexibility needed to address trainees with a wide range of experience and knowledge, tailoring, in a much finer way, the degree and type of support given, and, at the same time, spared us the exhaustive monitoring and interpretation of student's errors during an extended period, which would be required by alternative methods.

Nevertheless, it was found the need for a meta-knowledge layer in order to adapt the CBM method to an essentially procedural, time-dependent domain like the power system restoration field. In fact, the validity of certain constraints may be limited to only parts of the restoration process. On the other hand, the violation of a constraint can, in certain cases, render irrelevant the future verification of other constraints. Finally, equally valid constraints in a certain state of the process can have different relative importance from the didactic point of view. This fact suggests the convenience of establishing a constraint hierarchy.

This meta-knowledge layer is composed of rules that control the constraints' application, depending on several issues: the phase of the restoration process in which the trainee is; the constraints previously satisfied; and the set of constraints triggered simultaneously.

These rules establish a dependency network between constraints that can be represented by a graph (Fig. 3). The nodes (1-15) represent constraints. The relationships between constraints expressed by this graph can be of precedence, mutual exclusion or priority.

This tutoring module is able to train individual operators as if they were in a team, surrounded by virtual "operators", but is also capable of dealing with the interaction between several trainees engaged in a cooperative process. It provides specialized agents to fulfill the roles of the missing operators and, at the same time, monitors the cooperative work, stepping in when a serious imbalance is detected. The tutor can be used as a distance learning tool, with several operators being trained at different locations. 


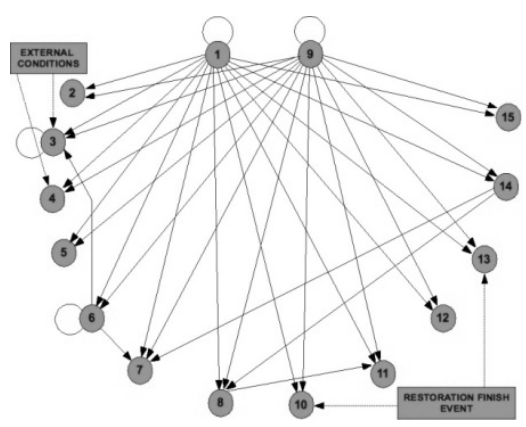

Fig. 3. Constraint Dependency Graph

\section{Modelling Power Systems Control Centre Operators According the New Reality}

Several acquisition methods and representation techniques have been proposed during the last decades to tackle the problem of how to represent the knowledge describing the student characteristics and preferences. Most of this work has been done to handle academic tutoring studies (e.g. training students on Mathematics, Physics or Programming). On-the-job training is a completely different matter. Trainees are also employees with very well defined tasks and responsibilities like to guarantee the effective operation of Power Systems networks in the case of CC Operators. So Power Systems CC Operators cannot be modeled like traditional trainees. For example, if an operator is being trained on-the-job, and some critical incident occurs he will need to stop training and pay attention to this new incident. This reality is always in the back of the mind of the Operators so training will need to be intelligent enough to catch the operators' model according these conditions.

With the expected evolution for Intelligent SCADA systems, more decentralized, flexible, and context-oriented, CC Operators will need to adopt a different level of skills (e.g. understanding when an incident affect areas strongly dependent of renewable sources). This means to introduce a semantic level above the SCADA system. The operator should work on the top of this new level.

Beyond the information about the trainee knowledge, the user model must trace the trainee misconceptions and knowledge about his actions during problem solving and the corresponding Tutor's feedback. This kind of knowledge will be maintained in the operator's historic training.

Another issue is the identification of a set of general factors concerning the trainee, such as acquisition and retention. Prior research indicates that examining general factors, like those referred above, can be useful to operator modeling. We also want to find how these general factors can be predictive of overall learning and contribute to improve the Tutor responses to the particular idiosyncrasies of the operator.

Finally we need to keep in mind the collaborative nature of Operators work, namely when critical incidents are occurring. In our previous work considering Incident Analysis and Diagnosis we considered the Operator alone and we treated this 
problem as being different from the Power and Service Restoration. Now, due to the increased complexity of Power Systems derived from the need to operate in a Competitive Electricity Market with Distributed Generation, Incident Analysis and Diagnosis will need to involve more people. A better combination with Power Generation companies, Transmission companies, and Distribution companies is much more necessary than in the past.

This means that we cannot model an operator alone. We will need to model a community of operators, working in the same or different companies, cooperating to maintain the Power System working effectively.

So, for this task it is necessary the development of a completely new model of collaborative communities of trainees. Notice that this is completely different from previous works in other areas, with cooperative students, since they are supposed to work in an academic environment and they are training on the same skills.

\section{Conclusions}

This paper described how Power Systems Control Center operators can be trained in two main tasks: Incident Analysis and Diagnosis; and Service Restoration. We gave some insights about the use of Cyber-Physical Systems and Ambient Intelligence concepts for this purpose, namely in which concerns the new reality of Electricity Markets, Renewable Sources and Distributed Generation. We explained how things change in tasks like the acquisition of domain knowledge on Incident Analysis, Diagnosis, and Power and Service Restoration, and in the modeling of Power Systems CC Operators according the new reality.

ITS is the approach we are using, combining several Artificial Intelligence techniques, namely: Multi-Agent Systems, Neural Networks, Constraint-based Modeling, Intelligent Planning, Knowledge Representation, Expert Systems, User Modeling, and Intelligent User Interfaces.

Concerning the operators' training, the most interesting features are:

1. The connection with SPARSE, a legacy Expert System used for Intelligent Alarm Processing [1].

2. The use of prediction tables and different granularity levels for fault diagnosis training.

3. The use of the model tracing technique to capture the operator's reasoning.

4. The development of two tools to help the adaptation of the curriculum to the opera-tor - one that generates training scenarios from real cases and another that assists in creating new scenarios.

5. The automatic assignment of the difficulty level to the problems.

6. The identification of the operators' knowledge acquisition factors.

7. The automatic selection of the next problem to be presented, using Neural Net-works.

8. The use of Multi-Agent Systems paradigm to model the interaction of several operators during system restoration. 
9. The use of the Constraint-based Modeling technique in restoration training.

10. The availability of an Intelligent User Interface in the interaction with the operator.

\section{References}

1. Vale, Z., Machado e Moura, A., Fernanda Fernandes, M., Marques, A., Rosado, C., Ramos, C.: SPARSE: An Intelligent Alarm Processor and Operator Assistant for Portuguese Substations Control Centers. IEEE Expert - Intelligent Systems and Their Application 12(3), 86-93 (1997)

2. Ramos, C., Augusto, J.C., Shapiro, D.: Ambient Intelligence: the next step for AI. IEEE Intelligent Systems magazine 23(2), 15-18 (2008)

3. Faria, L., Silva, A., Vale, Z., Marques, A.: Training Control Centres' Operators in Incident Diagnosis and Power Restoration Using Intelligent Tutoring Systems. IEEE Transactions on Learning Technologies (2009)

4. Lee, E.: Cyber Physical Systems: Design Challenges, University of California, Berkeley Technical Report No. UCB/EECS-2008-8

5. CPS Steering Group, Cyber-Physical Systems Executive Summary (2008), http://varma.ece.cmu .edu/Summit/CPS-Executive-Summary.pdf

6. Vale, Z., Morais, H., Silva, M., Ramos, C.: Towards a future SCADA. In: IEEE Power and Energy Society General Meeting General Meeting, Calgary, Alberta, Canada (2009)

7. Augusto, J.C., Cullagh, P.M.: Ambient Intelligence: Concepts and Applications. International Journal on Computer Science and Information Systems 4(1), 1-28 (2007)

8. ISTAG, Strategic Orientations \& Priorities for IST in FP6, European Commission Report (2002)

9. Ramos, C.: Ambient Intelligence Environments. In: Rabuñal, J., Dorado, J., Sierra, A. (eds.) Encyclopedia of Artificial Intelligence, Information Science Reference, pp. 92-98 (2009) ISBN 978-1-59904-849-9

10. Wenger, E.: Artificial intelligence and tutoring systems: computational and cognitive approaches to the communication of knowledge. Morgan Kaufmann Publishers Inc., San Francisco (1987)

11. Heffernan, N.T., Turner, T.E., Lourenco, A.L.N., Macasek, M.A., Nuzzo-Jones, G., Koedinger, K.R.: The ASSISTment Builder: Towards an Analysis of Cost Effectiveness of ITS Creation. In: Proceedings of the 19th International Florida Artificial Intelligence, Research Society Conference, pp. 515-520 (2006)

12. Aleven, V., Koedinger, K.R.: An effective meta-cognitive strategy: learning by doing and explaining with a computer-based Cognitive Tutor. Cognitive Science 26(2), 147-179 (2002)

13. Ramos, C., Frasson, C., Ramachandran, S.: Introduction to the Special Issue on Real World Applications of Intelligent Tutoring Systems. IEEE Transactions on Learning Technologies 2(2), 62-63 (2009) doi:10.1109/TLT.2009.16

14. Praça, I., Ramos, C., Vale, Z., Cordeiro, M.: MASCEM: A Multi-Agent Systems that simulates Competitive Electricity Markets. IEEE Intelligent Systems 18(6), 54-60 (2003)

15. Anderson, J., Corbett, A., Koedinger, K., Pelletier, R.: Cognitive Tutors: Lessons Learned. The Journal of the Learning Sciences 4(2), 167-207 (1995)

16. Stottler, R., Panichas, S.: A New Generation of Tactical Action Officer Intelligent Tutoring System (ITS). In: Proc. Industry/Interservice, Training, Simulation and Education Conference, I/ITSEC (2006) 
17. Sforna, M., Bertanza, V.: Restoration Testing and Training in Italian ISO. IEEE Transactions on Power Systems 17(4) (November 2002)

18. Jennings, N., Wooldridge, M.: Applying agent technology. Applied Artificial Intelligence: An International Journal 9(4), 351-361 (1995)

19. Ohlsson, S.: Constraint-Based Student Modeling. In: Greer, McCalla (eds.) Student Modeling: the Key to Individualized Knowledge-based Instruction, pp. 167-189. Springer, Heidelberg (1993)

20. Mitrovic, T., Koedinger, K., Martin, B.: A comparative analysis of Cognitive Tutoring and Constraint-based Modeling. In: em Brusilovsky, P., Corbett, A., Rovis, F. (eds.) 9th International Conference on User Modelling UM 2003. Springer, Heidelberg (2003) 